\title{
Bulk cohesive energy and surface tension from the size-dependent evaporation study of nanoparticles
}

\author{
K. K. Nanda $a^{a)}$ \\ Materials Research Centre, Indian Institute of Science, Bangalore-560 012, India
}

(Received 28 March 2005; accepted 1 June 2005; published online 8 July 2005)

In this letter, it is shown that the bulk cohesive energy $\left(E_{B}\right)$ and bulk surface tension $(\gamma)$ can be
deduced from the size-dependent evaporation (SDE) study of free nanoparticles. The surface tension
of nanoparticles $\left(\gamma_{n}\right)$ is obtained by analyzing SDE data on the basis of the Kelvin equation, while
$E_{B}$ is obtained by reanalyzing the SDE data. By comparing the size-dependent melting and the SDE
of nanoparticles, $\gamma$ is evaluated. @ 2005 American Institute of Physics. [DOI: $10.1063 / 1.1994958$ ]

Surface tension $(\gamma)$ is one of the important physical quantities that controls the growth of a material on a substrate as well as different phenomena, such as melting, coalescence, evaporation, phase transition, growth, etc., of nanoparticles. Similarly, cohesive energy $\left(E_{B}\right)$ is also one of the important physical quantities and is a measure of the thermal stability of the materials. Surface tension of solids is determined with contact angle method for which liquids with known surface tension are taken as a reference. ${ }^{1}$ On the other hand, $E_{B}$ is evaluated ${ }^{2}$ from the heat of sublimation $\left(\Delta H_{\mathrm{sub}}\right)$. In this letter, we evaluate $E_{B}$ and $\gamma$ of $\mathrm{Au}, \mathrm{Ag}$, and $\mathrm{PbS}$ from their size-dependent evaporation (SDE) data and conclude that the method can be employed to other systems as well. The SDE data ${ }^{3-5}$ are obtained by monitoring the onset temperature of evaporation $\left(T_{\text {onset }}\right)$ as a function of particle size (d) where $T_{\text {onset }}$ is the temperature at which the particle size (d) decreases as a consequence of the partial evaporation. Recently, we have shown that surface tension of nanoparticles $\left(\gamma_{n}\right)$ and the onset temperature of evaporation $\left(T_{\text {onset, } b}\right)$ of large particles can be obtained from the SDE data by verifying the Kelvin effect. ${ }^{3-5}$ Here, we show that $E_{B}$ can be evaluated by reanalyzing the same SDE data. Further, comparing our experimental results on SDE with the expression for the size-dependent melting (SDM), ${ }^{6}$ the variation of SDM and SDE is shown to be identical which establishes a correlation between $\gamma_{n}$ and $\gamma$. As a consequence, $\gamma$ can be evaluated directly if $\gamma_{n}$ is known and vice versa. Similarly, SDM can be predicted if SDE is known and vice versa.

Apart from the SDE study, $\gamma_{n}$ can also be deduced from the size-dependent lattice parameter (SDLP) of nanoparticles. $^{7-17}$ Different theoretical models predict a variation of $\gamma$ with $d$ for very small particles $(d \sim 0.1 \mathrm{~nm}){ }^{3}$ while $\gamma_{n}$ is almost size independent in the intermediate size range $(d>1.0 \mathrm{~nm})$. As the SDE and SDLP data are available in the intermediate size range, a discussion on $\gamma_{n}$ for very small particles is beyond our scope. Values of $\gamma_{n}$ reported for the $\mathrm{Ag}$ range ${ }^{7-12}$ from 1.415 to $6.56 \mathrm{~J} / \mathrm{m}^{2}$ and that for the $\mathrm{Au}$ range ${ }^{13-16}$ from 1.175 to $7.7 \mathrm{~J} / \mathrm{m}^{2}$. Alivisatos and his co-workers ${ }^{17}$ have obtained $\gamma_{n}=2.5 \mathrm{~J} / \mathrm{m}^{2}$ for bare CdS nanoparticles and $\gamma_{n}=1.74 \mathrm{~J} / \mathrm{m}^{2}$ for capped CdS nanoparticles. Thus, the difference in $\gamma_{n}$ for $\mathrm{Au}$ and $\mathrm{Ag}$ is believed to be due to the different surroundings and/or the different particle-substrate interaction. Further, any difference in the compressibility of nanoparticles and bulk, will affect the de-

\footnotetext{
${ }^{a)}$ Electronic mail: nanda@mrc.iisc.ernet.in
}

termination of $\gamma_{n}$. On the other hand, $\gamma_{n}$ is the only free parameter in the Kelvin equation and there is no ambiguity in evaluating $\gamma_{n}$ from the SDE data of nanoparticles. We also use SDE data ${ }^{3-5}$ of free nanoparticles which implies that there is no influence of substrates and/or the surroundings.

According to the Kelvin equation, the vapor pressure $\left(p_{s}\right)$ of nanoparticles is related to that of a flat surface $\left(p_{s 0}\right)$ as: ${ }^{18}$

$$
\frac{p_{s}}{p_{s 0}}=\exp \left(\frac{4 \gamma_{n} M}{\rho_{p} R T d}\right),
$$

where $M$ is the molecular weight, $\rho_{p}$ is the particle density, $R$ is the gas constant, $T$ is the temperature, and $d$ is the particle diameter. For Wulf crystals, $\gamma_{n} / d$ is constant for all crystal faces even though the surface energy is different for different crystal faces, which implies that Eq. (1) holds for all crystal faces of a nanoparticle. The vapor pressure $\left(p_{s 0}\right)$ can be related to $\Delta H_{\text {sub }}$ by the Clausius-Clapeyron equation as:

$$
\ln p_{s 0}=-\frac{\Delta H_{\text {sub }}}{R T}+C,
$$

where $R$ is the gas constant. Similar to bulk, the vapor pressure $\left(p_{s}\right)$ of nanoparticles can be related to its heat of sublimation $\left(\Delta H_{\text {sub }, n}\right)$ as

$$
\ln p_{s}=-\frac{\Delta H_{\mathrm{sub}, n}}{R T}+C .
$$

Substituting Eqs. (2) and (3) in Eq. (1), we obtain

$$
\Delta H_{\text {sub }, n}=\Delta H_{\text {sub }}-\frac{4 M \gamma_{n}}{\rho_{p} d} \Rightarrow \frac{\Delta H_{\text {sub }, n}}{\Delta H_{\text {sub }}}=1-\frac{4 v_{0} \gamma_{n}}{\Delta H_{\text {sub }} d} .
$$

In this context, it may be noted that $E_{B}, \Delta H_{\text {sub }}$, and the activation energy $\left(E_{A}\right)$ for bulk are comparable and can be realized by comparing these quantities. ${ }^{2}$ Here, we show that Eq. (4) can also be obtained from the total binding energy, $E(N)$ of nanoparticles, which can be expressed as

$$
E(N)=E_{B} N-4 \pi R^{2} \gamma_{n}=E_{B} N-4 \pi r_{s}^{2} \gamma_{n} N^{2 / 3},
$$

where $r_{s}$ is the radius of a sphere corresponding to the volume of one atom in bulk and $N$ is the number of atoms in the nanoparticles related to the particle radius as $R=r_{s} N^{1 / 3}$. Based on Eq. (5), $E_{A n}$ can be expressed as 

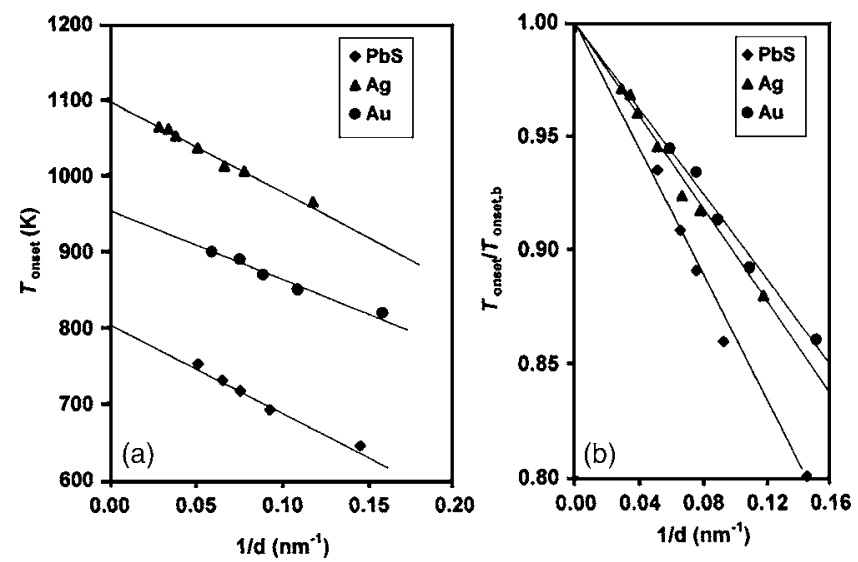

FIG. 1. (a) $T_{\text {onset }} \mathrm{vs} 1 / d$ for $\mathrm{PbS}, \mathrm{Ag}$, and Au. The data are taken from Refs. 3-5. (b) The same data are plotted as $T_{\text {onset }} / T_{\text {onset, b }}$ as a function of the inverse of particle size.

$$
E_{A n}=\frac{\partial E(N)}{\partial N}=E_{B}-\frac{8 \pi}{3} r_{s}^{2} \gamma_{n} N^{-1 / 3} \Rightarrow \frac{E_{A n}}{E_{B}}=1-\frac{8 \pi \gamma_{n} r_{s}^{2}}{3 E_{B} N^{1 / 3}}
$$

It may be noted from Eq. (6) that $E_{A}=E_{B}$ for large $N$ and Eq. (4) is same as Eq. (6). According the Trouton's rule, ${ }^{19}$ the ratio of latent heat of vaporization $\left(\Delta H_{\mathrm{vap}}\right)$ to the normal boiling point $\left(T_{b}\right)$ is constant. It may be noted that $E_{A n}$ is the energy required to remove one atom from the nanoparticle, while $T_{\text {onset }}$ is the temperature at which the atoms/molecules leave the particles and as a consequence the particle size decreases. This indicates that $E_{A n}$ can be scaled to $T_{\text {onset }}$ and $E_{A}$ can be scaled to $T_{\text {onset }, b}$. Equation (6) can then be expressed as

$$
\frac{T_{\text {onset }}}{T_{\text {onset, } \mathrm{b}}}=1-\frac{16 \pi \gamma_{n} r_{s}^{3}}{3 E_{B} d} .
$$

Equation (7) predicts a linear relation between $T_{\text {onset }}$ and the inverse of the particle size and the slope depends on $\gamma_{n}$ and $E_{B}$. It may be noted that $E_{B}$ can easily be evaluated from the slope obtained by plotting $T_{\text {onset }} / T_{\text {onset }, b}$ as a function of $1 / d$, if $\gamma_{n}$ is known. It has been shown both experimentally and theoretically (based on the Kelvin equation) that $T_{\text {onset }}$ is linear with respect to $1 / d$ and the slope depends on $\gamma_{n} \cdot{ }^{3-5}$ Analyzing the SDE data on the basis of Kelvin equation, $\gamma_{n}$ for $\mathrm{PbS}, \mathrm{Ag}$, and $\mathrm{Au}$ has been found to be 2.45, 7.2, and $8.78 \mathrm{~J} / \mathrm{m}^{2}$, respectively. ${ }^{3-5}$ To deduce $E_{B}$ from the SDE data, the data ${ }^{3-5}$ of $\mathrm{PbS}, \mathrm{Ag}$, and Au nanoparticles are plotted as $T_{\text {onset }}$ versus $1 / d$ and is shown in Fig. 1(a). The least-squares fit of the experimental data into a straight line, yields a slope of $-1138 \pm 75,-1158 \pm 55,-895 \pm 25 \mathrm{~nm} \mathrm{~K}$, and an intercept of $805 \pm 8,1097 \pm 4,953 \pm 4 \mathrm{~K}$ for $\mathrm{PbS}, \mathrm{Ag}$, and $\mathrm{Au}$, respec- tively. It may be noted that the intercept represents a temperature $T_{\text {onset, } b}$ where large particles evaporate under the experimental conditions used to study the SDE. The lower value of $T_{\text {onset, } b}$ for $\mathrm{Au}$ is believed to be due to surface melting. ${ }^{5}$ Now, we plot SDE data as $T_{\text {onset }} / T_{\text {onset }, b}$ versus $1 / d$ and shown in Fig. 1(b). The slopes are found to be $-1.41 \pm 0.11,-1.05 \pm 0.05$, and $-0.94 \pm 0.03 \mathrm{~nm}$, respectively, for $\mathrm{PbS}, \mathrm{Ag}$, and $\mathrm{Au}$. Taking $r_{s}=0.158 \mathrm{~nm}$ and $\gamma_{n}$ $=8.97 \mathrm{~J} / \mathrm{m}^{2}, E_{B}$ of $3.88 \pm 0.09 \mathrm{eV}$ is obtained for Au. Similarly, $E_{B}$ of $2.88 \pm 0.12$ and $2.33 \pm 0.18 \mathrm{eV}$ are obtained for $\mathrm{Ag}$ and $\mathrm{PbS}$ from their SDE data. ${ }^{3,4} E_{B}$ of $\mathrm{Au}$ and $\mathrm{Ag}$ are in excellent agreement with the experimental values (Table I) of 3.81 and $2.95 \mathrm{eV}$, respectively. ${ }^{20}$ By fitting the vapor pressure data $^{21}$ of $\mathrm{PbS}$ in the temperature range of $1028-1381 \mathrm{~K}$, $\Delta H_{\text {sub }}$ is determined as $234.4 \mathrm{~kJ} / \mathrm{mole}(2.43 \mathrm{eV} /$ atom $)$. It may be noted that $\mathrm{PbS}$ evaporates ${ }^{22}$ in a molecular form and $\Delta H_{\text {sub }}$ can be considered to be equal to $E_{B}$ which is in excellent agreement with that obtained from SDE data. Overall, it can be concluded that the cohesive energy per atom can be evaluated from the SDE data.

It is well established both experimentally and theoretically that the melting temperature $\left(T_{m}\right)$ of nearly free nanoparticles drops linearly with the inverse of the particle size as is the case of SDE. Based on the experimental results (Fig. 1) on SDE, the slope of $T_{\text {onset }} / T_{\text {onset }, b}$ is $-1.41,-1.05$, and $-0.94 \mathrm{~nm}$ for $\mathrm{PbS}, \mathrm{Ag}$, and $\mathrm{Au}$, respectively. On the other hand, the slope of $T_{m} / T_{m b}$ versus $1 / d$ curve is reported to be in the range from -2.0 to $-0.8 \mathrm{~nm}$ for $\mathrm{Au},{ }^{23-26}$ while most of the theoretical models predict the slope as close to $-1.0 \mathrm{~nm} .^{6,23,26-28}$ Similarly, the slope is found to be about $-2.0 \mathrm{~nm}$ from the experimental data of $\mathrm{Ag}^{26}$ while the molecular dynamics simulations ${ }^{29}$ and other theoretical models ${ }^{6,28}$ predict a slope of about -0.7 and $-1.0 \mathrm{~nm}$, respectively. As per the expression for SDM based on the liquiddrop model, ${ }^{6}$ the slope of $T_{m} / T_{m b}$ versus $1 / d$ curve is -0.97 and $-1.13 \mathrm{~nm}$ for $\mathrm{Ag}$ and $\mathrm{Au}$, respectively, and is nearly the same as that obtained from $T_{\text {onset }} / T_{\text {onset, } b}$ versus $1 / d$. This indicates that the variation of $T_{\text {onset }} / T_{\text {onset }, b}$ and $T_{m} / T_{m b}$ can be assumed to be identical. However, SDE data yield $\gamma_{n}$ $=7.20$ and $8.78 \mathrm{~J} / \mathrm{m}^{2}$ for $\mathrm{Ag}$ and $\mathrm{Au}$, respectively, ${ }^{4,5}$ while $\gamma$ of 1.065 and $1.363 \mathrm{~J} / \mathrm{m}^{2}$ is used ${ }^{6}$ to predict the SDM of $\mathrm{Ag}$ and Au nanoparticles, respectively. Further, cohesive energy per atom is obtained from the SDE data, whereas cohesive energy per coordination ${ }^{6}$ is used to evaluate SDM. The cohesive energy per coordination is six times lower than the cohesive energy per atom for $\mathrm{Ag}$ and $\mathrm{Au}$. This is because the coordination number is 12 for $\mathrm{Ag}$ and $\mathrm{Au}$ (face-centeredcubic structure) and each bond is shared by two atoms. It may also be noted that $\gamma_{n}$ obtained from the SDE data is approximately six times higher than $\gamma$ for Ag and Au. This indicates that $\gamma_{n}$ is related to $\gamma$ in the same way as the co-

\begin{tabular}{|c|c|c|c|c|c|c|c|}
\hline \multirow[t]{2}{*}{ Materials } & \multicolumn{2}{|c|}{ Slope (nm) } & \multirow[t]{2}{*}{$\begin{array}{c}\gamma_{n}\left(\mathrm{~J} / \mathrm{m}^{2}\right) \\
(\text { Our work })\end{array}$} & \multicolumn{2}{|c|}{$\gamma\left(\mathrm{J} / \mathrm{m}^{2}\right)$} & \multicolumn{2}{|c|}{$E_{B}(\mathrm{eV} /$ atom $)$} \\
\hline & $T_{\text {onset }} / T_{\text {onset }, b}$ & $\begin{array}{l}T_{m} / T_{m b} \\
\text { (Ref. 6) }\end{array}$ & & (Our work) & (Ref. 27) & (Our work) & (Ref. 20) \\
\hline $\mathrm{PbS}$ & -1.41 & $\bullet \bullet$ & 2.45 & 0.82 & $\sim 1.0^{\mathrm{a}}$ & 2.33 & $2.43^{\mathrm{a}}$ \\
\hline $\mathrm{Ag}$ & -1.05 & -0.97 & 7.20 & 1.20 & $1.1-1.2$ & 2.88 & 2.95 \\
\hline $\mathrm{Au}$ & -0.94 & -1.13 & 8.78 & 1.46 & $1.2-1.4$ & 3.88 & 3.81 \\
\hline
\end{tabular}

TABLE I. Different parameters obtained from the SDE data. 
hesive energy per atom is related to the cohesive energy per coordination and $\gamma$ can be evaluated directly if $\gamma_{n}$ is known. A similar relation between $\gamma_{n}$ and $\gamma$ has also been obtained by analyzing the experimental data on the cohesive energy of Mo and $\mathrm{W}$ nanoparticles based on the liquid-drop model (to be published elsewhere).

As the coordination number of materials with bodycentered-cubic, simple cubic, and diamondlike crystal structure is 8,6 , and 4 , respectively, $\gamma_{n}$ will be four, three, and two times higher than $\gamma$. The crystal structure of $\mathrm{PbS}$ is $\mathrm{NaCl}$ type and hence, the coordination number is 6 that yields $\gamma$ $=0.82 \mathrm{~J} / \mathrm{m}^{2}$. This value seems to be realistic as $\mathrm{PbS}$ is an ionic semiconductor and $\gamma$ of an ionic semiconductor is $\sim 1.0 \mathrm{~J} / \mathrm{m}^{2}$.

In summary, it is shown that SDE data can be used to evaluate $\gamma_{n}, \gamma$, and $E_{B}$ and the variation of $T_{m}$ and $T_{\text {onset }}$ is almost identical. As $\gamma_{n}$ is evaluated and then used to determine $E_{B}$ from the same data, $E_{B}$ is believed to be independent of the influence of substrates or surroundings. However, as $\gamma_{n}$ is influenced by the surroundings, the value obtained for free nanoparticles should be used to evaluate $\gamma$. Though the analysis of the SDE and SDM data elucidates a relation between $\gamma$ and $\gamma_{n}$, investigation of elements with different crystal structure is required to confirm the validity of the relationship.

The author acknowledges Dr. F. E. Kruis and Professor W. A. Harrison for valuable discussions.

${ }^{1}$ P. J. Sell and A. W. Neumann, Angew. Chem., Int. Ed. Engl. 5, 299 (1965).

${ }^{2}$ B. Farid and R. W. Godby, Phys. Rev. B 43, 14248 (1991), and references therein.

${ }^{3}$ K. K. Nanda, F. E. Kruis, and H. Fissan, Phys. Rev. Lett. 89, 256103 (2002).

${ }^{4}$ K. K. Nanda. A. Maisels, F. E. Kruis, H. Fissan, and S. Stappert, Phys.
Rev. Lett. 91, 106102 (2003).

${ }^{5}$ K. K. Nanda, A. Maisels, F. E. Kruis, H. Fissan, and B. Rellinghaus (unpublished).

${ }^{6}$ K. K. Nanda, S. N. Sahu, and S. N. Behera, Phys. Rev. A 66, 013208 (2002).

${ }^{7}$ C. R. Berry, Phys. Rev. 88, 596 (1952).

${ }^{8}$ T. de Planta, R. Ghez, and F. Piuz, Helv. Phys. Acta 37, 74 (1964).

${ }^{9}$ H. J. Wasserman and J. S. Vermaak, Surf. Sci. 22, 164 (1970).

${ }^{10}$ P. A. Montano, W. Schulze, B. Tesche, G. K. Shenoy, and T. I. Morrison, Phys. Rev. B 30, 672 (1984).

${ }^{11}$ H. Hofmeister, S. Thiel, M. Dubiel, and E. Schurig, Appl. Phys. Lett. 70, 1694 (1997).

${ }^{12}$ F. Piuz, Helv. Phys. Acta 37, 620 (1964).

${ }^{13}$ C. W. Mays, J. S. Vermak, and D. Kuhlmann-Wilsdorf, Surf. Sci. 12, 134 (1968).

${ }^{14}$ C. Solliard and M. Flueli, Surf. Sci. 156, 487 (1985).

${ }^{15}$ R. Lamber, S. Wetjen, G. Schulz-Ekloff, and A. Baalmann, J. Phys. Chem. 99, 13834 (1995).

${ }^{16}$ P. D. Cluskey, R. J. Newport, R. E. Benfield, S. J. Gurman, and G. Schmid, Z. Phys. D: At., Mol. Clusters 26, 8 (1993).

${ }^{17}$ A. N. Goldstein, C. M. Echer, and A. P. Alivisatos, Science 256, 1425 (1992).

${ }^{18}$ W. Thomson (Kelvin), Philos. Mag. 42, 448 (1871).

${ }^{19}$ F. T. Trouton, Philos. Mag. 18, 54 (1884).

${ }^{20}$ C. Kittel, Introduction to Solid State Physics, 5th ed. (Wiley, New York, 1976).

${ }^{21}$ L. Gmelin, in Gmelin Handbook of Inorganic Chemistry, edited by E. H. E. Pietsch (Springer, Berlin, 1979).

${ }^{22}$ S. K. Elrahaiby and Y. K. Rao, Metall. Trans. B 13, 633 (1992).

${ }^{23}$ P. Buffat and J.-P. Borel, Phys. Rev. A 13, 2287 (1976).

${ }^{24}$ K. Dick, T. Dhanasekaran, Z. Zhang, and D. Meisel, J. Am. Chem. Soc. 124, 2312 (2002).

${ }^{25}$ J. R. Sambles, Proc. R. Soc. London, Ser. A 324, 339 (1971).

${ }^{26}$ T. Castro, R. Reifenberger, E. Choi, and R. P. Andres, Phys. Rev. B 42, 8548 (1990).

${ }^{27}$ S. Arcidiacono, N. R. Bieri, D. Poulikakos, and C. P. Grigoropoulos, Int. J. Multiphase Flow 30, 979 (2004).

${ }^{28}$ M. Wautelet, J. Phys. D 24, 343 (1991).

${ }^{29}$ S. J. Zhao, S. Q. Wang, Z. Q. Yang, and H. Q. Ye, J. Phys.: Condens. Matter 13, 8061 (2001). 